\title{
O uso de situações reais como promotor de aprendizagem significativa no âmbito de
}

\section{sistemas de informação}

The use of real situations as a promoter of meaningful learning in the scope of information systems

El uso de situaciones reales como promotor de aprendizajes significativos en el ámbito de los sistemas de información

Recebido: 25/01/2021 | Revisado: 31/01/2021 | Aceito: 03/02/2021 | Publicado: 08/02/2021

\author{
Cristiano Tolfo \\ ORCID: https://orcid.org/0000-0002-1950-6461 \\ Universidade Federal do Pampa, Brasil \\ E-mail: cristianotolfo@unipampa.edu.br \\ Felipe Antunes Quirino \\ ORCID: https://orcid.org/0000-0002-4439-9450 \\ Universidade Federal do Pampa, Brasil \\ E-mail: felipeantunesquirino@gmail.com
}

\begin{abstract}
Resumo
A promoção de aprendizagem ativa que associe teoria e prática de forma a resultar na construção autônoma do conhecimento é um dos desafios presentes no atual contexto do ensino superior. O objetivo deste trabalho é analisar o uso de situações reais como promotor de aprendizagem significativa no âmbito de sistemas de informação. Com base em experiência vivenciada em uma disciplina de graduação no curso de ciência da computação, são analisados a estratégia de ensino e os recursos didáticos adotados. A estratégia de ensino objeto de estudo foi a análise de situaçõesproblema, enquanto que os recursos didáticos analisados foram um modelo de resolução de problemas em sistemas de informação e uma técnica de modelagem de processos de negócios. A estratégia de ensino e os recursos didáticos em questão, foram utilizados para analisar a aplicabilidade de uma situação-problema real. Verificou-se que a referida estratégia de ensino pode promover o aprendizado significativo. Foi verificado também que o uso dos recursos didáticos em questão, possibilita identificar não apenas questões tecnológicas de soluções de sistemas de informação, contemplando também questões humanas e organizacionais. O estudo aponta para o potencial de replicabilidade da estratégia e dos recursos didáticos para o ensino e a aprendizagem ativa em sistemas de informação e possibilita concluir sobre a importância do prévio planejamento de estratégias de ensino e a adequada escolha de recursos didáticos.

Palavras-chave: Ensino; Ensino superior; Aprendizagem significativa; Aprendizagem baseada em problemas; Sistemas de informação.
\end{abstract}

\begin{abstract}
The promotion of active learning that combines theory and practice in order to result in the autonomous construction of knowledge is one of the challenges present in the current context of higher education. The objective of this work is to analyze the use of real situations as a promoter of meaningful learning in the scope of information systems. Based on experience in an undergraduate course in the computer science course, the teaching strategy and teaching resources adopted are analyzed. The teaching strategy object of study was the analysis of problem situations, while the didactic resources analyzed were a problem-solving model in information systems and a business process modeling technique. The teaching strategy and didactic resources in question were used to analyze the applicability of a real problem situation. It was found that the said teaching strategy can promote meaningful learning. It was also verified that the use of the didactic resources in question, makes it possible to identify not only technological issues of information systems solutions, but also contemplating human and organizational issues. The study points to the potential for replicability of strategy and didactic resources for teaching and active learning in information systems and makes it possible to conclude on the importance of prior planning of teaching strategies and the appropriate choice of teaching resources.
\end{abstract}

Keywords: Teaching; Higher education; Meaningful learning; Problem-based learning; Information systems.

\section{Resumen}

La promoción de aprendizajes activos que combinen la teoría y la práctica para dar como resultado la construcción autónoma del conocimiento es uno de los desafíos presentes en el contexto actual de la educación superior. El objetivo de este trabajo es analizar el uso de situaciones reales como promotoras de aprendizajes significativos en el ámbito de los sistemas de información. A partir de la experiencia en un curso de pregrado de la carrera de informática, se analiza la estrategia didáctica y los recursos didácticos adoptados. La estrategia docente objeto de estudio fue el análisis de situaciones problemáticas, mientras que los recursos didácticos analizados fueron un modelo de resolución de problemas 
en sistemas de información y una técnica de modelado de procesos de negocio. La estrategia de enseñanza y los recursos didácticos en cuestión se utilizaron para analizar la aplicabilidad de una situación problemática real. Se encontró que dicha estrategia de enseñanza puede promover un aprendizaje significativo. También se verificó que el uso de los recursos didácticos en cuestión, permite identificar no solo cuestiones tecnológicas de soluciones de sistemas de información, contemplando también cuestiones humanas y organizativas. El estudio apunta al potencial de replicabilidad de los recursos estratégicos y didácticos para la enseñanza y el aprendizaje activo en los sistemas de información y permite concluir sobre la importancia de la planificación previa de las estrategias de enseñanza y la adecuada elección de los recursos didácticos.

Palabras clave: Enseñanza; Enseñanza superior; Aprendizaje significativo; Aprendizaje basado en problemas; Sistemas de información.

\section{Introdução}

Um dos grandes desafios que se impõem no ensino superior atualmente é a promoção de participação ativa e de aprendizagem significativa, de modo que os alunos consigam aliar teoria à prática de assuntos abordados em sala de aula. Neste sentido, se torna primordial o prévio planejamento das aulas, baseado em estratégias de ensino e uso de recursos didáticos adequados para cada componente curricular, considerando-se a ementa, os objetivos e o conteúdo programático (Tolfo, 2017a).

A estratégia de ministrar aulas expositivas e dialogadas é uma alternativa básica para disciplinas de qualquer área de conhecimento. Contudo, é preciso ter cautela para que a referida

Estratégia não conduza a situações em que as aulas se tornem monólogas, tendendo à monotonia. A respeito disso, Tolfo (2017a, p.11) afirma que:

No âmbito educacional, o ensino baseado predominantemente na transmissão de informações por meio de aulas expositivas e monologas, tem se tornando insatisfatório frente à grande disponibilidade e facilidade de acesso à informação que os alunos vivenciam fora da sala de aula. Da mesma forma, se torna questionável o aprendizado baseado exclusivamente na estratégia de aulas expositivas, em que o educador somente expõe conteúdos que precisam ser decorados pelos educandos, para que, posteriormente, sejam reproduzidos em uma avaliação.

A aprendizagem baseada em problemas (Araújo, \& Sastre, 2009; Gil, 2012; Tolfo, 2017b) e a aprendizagem significativa (Ausubel, 1982; Mancini, 2005; Moreira, 2010) são algumas das estratégias de ensino que promovem o papel protagonista do aluno na construção do seu próprio aprendizado. No que diz respeito aos recursos didáticos, existem as mais variadas possibilidades, cabendo ao docente identificar qual é a opção mais adequada para o contexto da disciplina e para o perfil da turma.

O objetivo deste trabalho é analisar o uso de situações reais como promotor de aprendizagem significativa no âmbito de sistemas de informação. Trata-se do relato de uma experiência de ensino e aprendizagem ativa no âmbito de sistemas de informação. Na referida experiência utilizou-se como recurso didático um modelo de resolução de problemas de sistemas de informação e uma técnica de modelagem de processos de negócios.

$\mathrm{O}$ relato contempla um trabalho acadêmico realizado em um componente curricular no ensino superior, no qual se adotou como estratégia de ensino a aprendizagem baseada em problemas para abordar assuntos relacionados à sistemas de informação. Como recurso didático foi adotado o modelo de resolução de problemas de sistemas de informação proposto por Laudon \& Laudon (2007) e a modelagem de processos de negócios utilizando a notação Business Process Model and Notation - BPMN (Omg, 2021).

\section{Sistemas de Informação}

De acordo com Baltzan e Philips (2012), os sistemas de informação auxiliam a tomada de decisão e a resolução de problemas no âmbito organizacional, bem como na identificação de oportunidades na esfera de negócios. Os referidos autores 
apontam como principais razões para o crescente uso de sistemas de informação a necessidade de: analisar grandes quantidades de informação gerada por tecnologias digitais; tomar decisão em tempo real; utilizar técnicas de análises preditivas e também proteger ativos informacionais.

A grande quantidade de informações geradas no atual cenário digital está associada aos fenômenos do Big Data (Davenport, 2014) e da transformação digital (Rogers, 2019) que atualmente determinam novas dinâmicas de trabalho e de relacionamento em praticamente todas as esferas produtivas e sociais. O uso generalizado de sistemas e de dispositivos móveis conectados à internet gera dados que precisam tornar-se informação. Isso determina uma dinâmica organizacional na qual adotar a perspectiva de sistemas de informação torna-se cada vez mais útil para auxiliar na tomada de decisão e na resolução de problemas, considerando não apenas questões técnicas, mas também aspectos humanos e organizacionais envolvidas nas possíveis soluções.

Os sistemas de informação gerenciais envolvem a coleta, o armazenamento e a distribuição de informações que apoiam a tomada de decisão e a resolução de problemas no âmbito organizacional. De acordo com Laudon e Laudon (2007, p.8):

Um sistema de informação pode ser definido tecnicamente como um conjunto de componentes inter-relacionados que coletam (ou recuperam), processam, armazenam e distribuem informações destinadas a apoiar a tomada de decisões, a coordenação e o controle de uma organização. Além de dar apoio à tomada de decisões, à coordenação e ao controle, esses sistemas também auxiliam os gerentes e trabalhadores a analisar problemas.

De acordo com Laudon e Laudon (2007), as informações processadas para auxiliar na tomada de decisão são oriundas de fontes internas e externas da organização. Já a resolução de problemas em sistemas de informação envolve a análise de dimensões humanas, organizacionais e tecnológicas. Ao analisar um problema em sistemas de informação, na dimensão humana, parte do problema pode estar relacionado à falta de motivação ou de conhecimento técnico da equipe. Na dimensão organizacional, parte do problema pode estar relacionado com processos ultrapassados e excessivamente burocratizados. $\mathrm{Na}$ dimensão humana, a falta de recursos tecnológicos apropriados pode caracterizar outra parte do problema. A respeito da resolução de problemas, Laudon e Laudon (2007) afirmam que:

Solucionar problemas é um processo contínuo de quatro passos. Durante e depois da implantação, o resultado precisa ser continuamente medido, e os solucionadores de problemas devem procurar saber em que medida a solução está funcionando. Desse modo, a identificação do problema pode mudar ao longo do tempo, as soluções podem ser alteradas e novas escolhas podem ser feitas, tudo com base na experiência (Laudon \& Laudon, 2007, p.16).

O modelo de resolução de problemas organizacionais sob a ótica de sistemas de informação proposto por Laudon e Laudon (2007) envolve as etapas de: identificação do problema, propostas de soluções, avaliação das propostas, escolha da solução e implantação da solução escolhida. Ressalta-se que o feedback se faz necessário nas demais etapas do modelo em questão.

\section{Modelagem de Processos de Negócios}

As organizações podem ser vistas como um conjunto de processos inter-relacionados que funcionam de forma ordenada para atender objetivos de negócio. A modelagem de processos de negócios é uma técnica amplamente utilizada para representar tais processos (Valle \& Oliveira, 2009; Pradella, Furtado \& Kipper, 2012; Tolfo, 2019).

A notação BPMN - Business Process Model and Notation (Omg, 2021) é uma forma padrão para representar processos de negócios. De acordo com Braconi e Oliveira (2009), essa técnica pode ser utilizada para representar os mais variados tipos de processos: 
Trata-se de uma técnica abrangente que oferece recursos para a modelagem dos mais variados tipos de processos, desde os mais genéricos aos específicos. Por isso, pode ser usada na modelagem de processos de qualquer natureza, como: administrativos (compras, vendas, controles de materiais, etc.), financeiros (empréstimos, aplicações, controle de capital, etc.), operacionais (manutenção, fabricação, distribuição, etc.), garantia da qualidade, desenvolvimento de software, desenvolvimento de produtos ou de serviços, etc. (Braconi \& Oliveira, 2009, p.78).

A ampla adoção da notação BPMN para a modelagem de processos ocorre pelo fato da mesma ser de uso intuitivo e possuir considerável capacidade de representação de fluxos informacionais e de trabalho.

A notação BPMN é composta por um conjunto de elementos básicos e avançados que possibilita representar processos em diferentes níveis de detalhamento. Contidos em um diagrama de processo, os elementos básicos de um processo envolvem eventos de início e de fim, tendo entre eles tarefas interligadas por fluxos de sequência e momentos de decisão representados por gateways. A Figura 1 ilustra parte dos elementos básicos da BPMN.

Figura 1. Elementos básicos da notação BPMN.

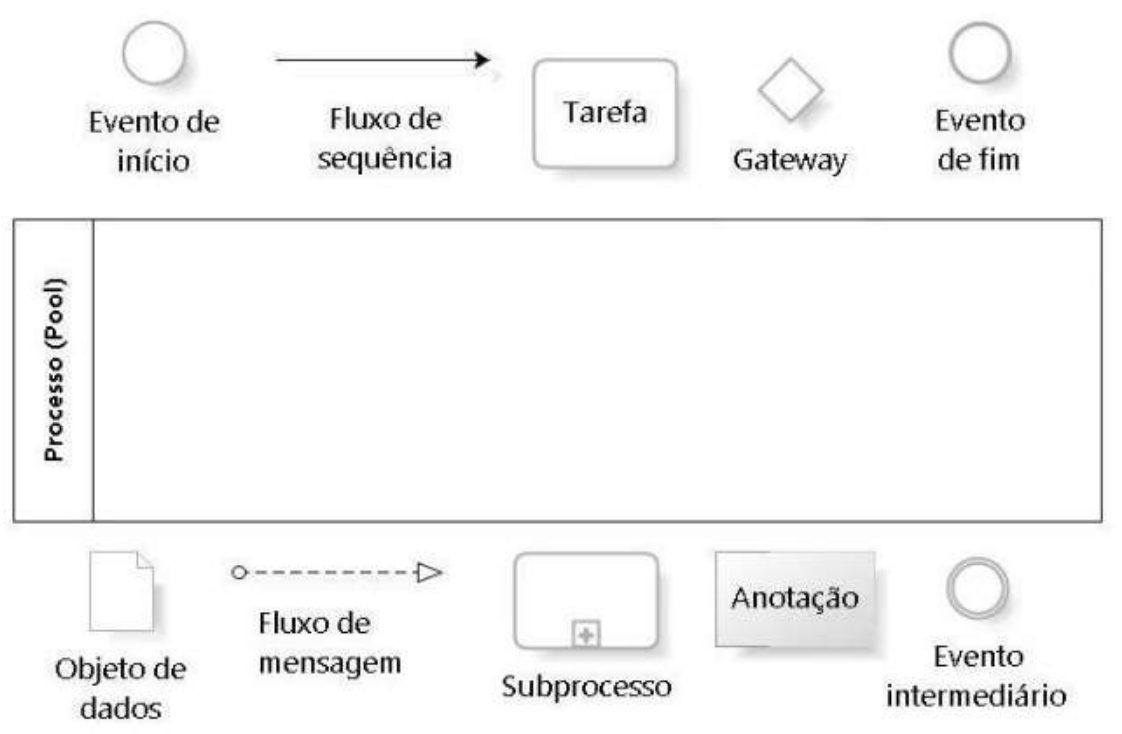

Fonte: Adaptado de Tolfo (2019, p.18)

Uma das técnicas de modelagem de processos com BPMN utilizadas para a análise e melhoria de processos é a técnica de modelar o AS-IS e o TO-BE de processos. A modelagem do AS-IS consiste em modelar o estado atual do processo, tal como ele vem sendo executado. A partir desta modelagem é possível analisar pontos de melhoria no mesmo.

As melhorias identificadas com a modelagem do AS-IS são representadas em uma nova versão do processo. Trata-se da modelagem do TO-BE do processo, que corresponde ao estado futuro do processo. Na modelagem do TO-BE é possível visualizar como será o estado futuro do processo, contemplando as mudanças idealizadas.

As técnicas de modelagem AS-IS e TO-BE podem ser aplicadas nas mais variadas situações em que se busca analisar processos, por exemplo, para otimizar processos excessivamente burocratizados, padronizar processos, informatizar e digitalizar processos manuais, documentar e comunicar processos que vêm ocorrendo de modo informal. Baldam et al. (2007); Baltzan e Phillips (2012); Tolfo, Medeiros, e Mombach (2013); Tolfo e Flora (2016) e Tolfo (2019) são alguns dos autores que abordam modelagem AS-IS e TO-BE.

\section{Metodologia}

No que diz respeito aos aspectos metodológicos do presente trabalho, observando as classificações realizadas por 
Silveira e Córdova (2009), pode-se afirmar que se trata de uma pesquisa com abordagem qualitativa, visto que contempla a análise de uma experiência vivenciada no ensino superior, sem foco em realizar argumentações baseadas em representatividade numérica. Quanto a natureza, trata-se de uma pesquisa aplicada, pois envolve a aplicação prática de estratégias de ensino e recursos didáticos em uma disciplina de sistemas de informação. No tocante aos objetivos e procedimentos da pesquisa, o estudo configura uma pesquisa descritiva que envolve um estudo de caso no qual são descritos fatos relacionados à experiência de ensino e de aprendizagem vivenciada pelos autores do trabalho.

Este trabalho envolve um relato de ensino e de aprendizagem sobre sistemas de informação. Apresenta-se uma experiência com a aplicação de um modelo de resolução de problemas de sistemas de informação, seguido da análise e modelagem de processos relacionados ao mesmo.

Este relato é baseado em uma experiência vivenciada com um trabalho acadêmico realizado por um aluno da disciplina Sistemas de Informação, que é um componente curricular do curso de Ciência da Computação da Universidade Federal do Pampa (Unipampa, 2019).

O trabalho acadêmico em questão teve como objeto de estudo uma luva de captação de movimentos, a qual vem sendo desenvolvida por um grupo de pesquisa da UNIPAMPA e por uma startup incubada no parque tecnológico da mesma universidade. Parte dos resultados obtidos com a análise da situação-problema, incluindo a luva de captação de movimentos, foi publicada por Quirino, Girardi e Tolfo (2020).

Para a análise da situação-problema em questão, utilizou-se o modelo de resolução de problemas de sistemas de informação proposto por Laudon e Laudon (2007). Buscou-se analisar questões humanas, organizacionais e tecnológicas que poderiam estar envolvidas na aplicação da luva de captação de gestos em clínicas que prestam serviços de fisioterapia.

Na sequência, utilizou-se a modelagem de processos de negócios para analisar como poderiam ocorrer os processos relacionados aos testes de motricidades de modo manual e com o auxílio da luva de captação de movimentos.

O trabalho relatado foi realizado durante a disciplina Sistemas de Informação, tendo sido adotada a técnica de modelar o estado atual do processo objeto de estudo, que representa a versão AS-IS do mesmo. Posteriormente, buscou-se identificar pontos de melhoria, os quais são representados em uma versão que contempla o estado futuro do processo, que é a versão TOBE. Na Figura 2 está descrito o processo adotado durante a disciplina para modelar cada uma das versões dos processos estudados. 
Figura 2. Processo de modelagem do AS-IS e do TO-BE.

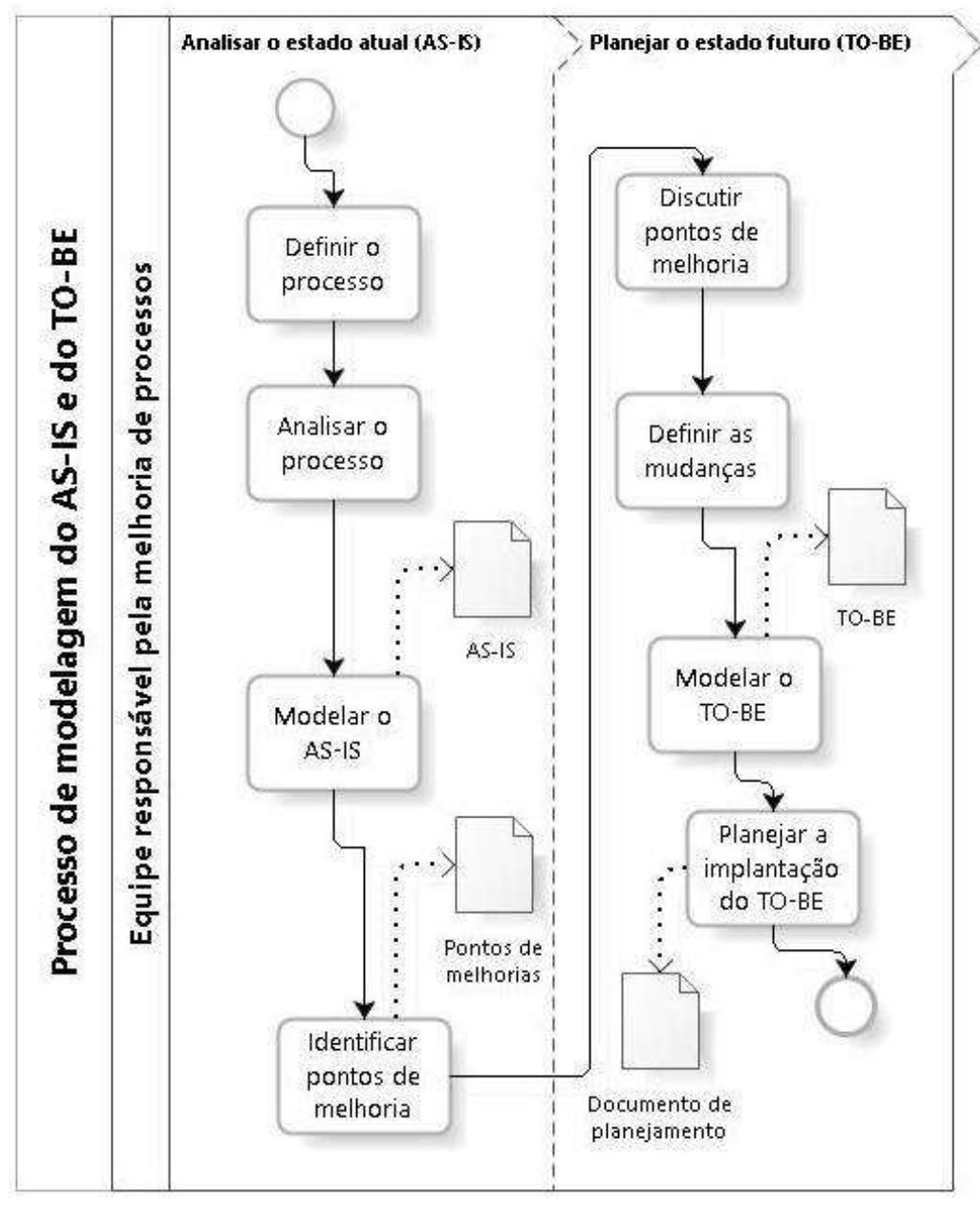

Fonte: Adaptado de Tolfo (2019).

Além de descrever parte dos procedimentos metodológicos adotados na disciplina Sistemas de Informação, o processo descrito na Figura 2 também contempla um exemplo de modelagem de processos com a notação BPMN. Para modelar o referido processo foi utilizada uma versão gratuita da ferramenta BizAgi Modeler (Bizagi, 2020). A BizAgi é específica para a modelagem de processos, podendo desenvolver soluções em diferentes níveis de modelagem, desde a descrição de fluxos de trabalho até o desenvolvimento de sistemas de workflow que automatizam processos. Cabe mencionar que existe também uma gama de ferramentas on-line para a modelagem de processos com diferentes níveis de recurso.

De acordo com Tolfo (2020, p.14), no âmbito do ensino superior “a abordagem AS-IS e TO-BE está alinhada à aprendizagem baseada em problemas, pois possibilita a análise de problemas reais e envolve os alunos de modo ativo na busca da solução para o problema".

Sob a perspectiva de sistemas de informação e da modelagem de processos, o trabalho acadêmico relatado neste artigo teve como objeto de estudo a solução tecnológica relacionada a uma luva para captação de movimentos que possibilita a realização de testes de motricidade ao captar movimentos de pessoas com alguma categoria de deficiência motora.

Trabalhos como os realizados por Baldissera, Quirino, Romanssini e Girardi, (2018); Quirino, Romanssini, Girardi e Baldissera (2018) e Romanssini, Quirino e Girardi (2018), abordam a solução e detalhes tecnológicos da luva de captação de movimentos em questão. 


\section{Resultados e Discussões}

Como parte dos resultados obtidos, apresenta-se a versão do modelo de resolução de problemas de sistemas de informação que contempla uma perspectiva de questões humanas, organizacionais e tecnológicas envolvidas na adoção da luva de captação de movimentos por estabelecimentos de tratamento fisioterapêutico.

Parte deste estudo foi publicado por Quirino, Girardi e Tolfo (2020), tendo sido utilizando o modelo de resolução de problemas de sistemas de informação proposto por Laudon e Laudon (2007). Na Figura 3 são listados possíveis desafios organizacionais em relação aos quais a luva de captação de movimentos poderia fazer parte da solução na dimensão tecnológica.

Figura 3. Modelo de resolução de problemas de sistemas de informação baseado na aplicação da luva de captação de movimentos.

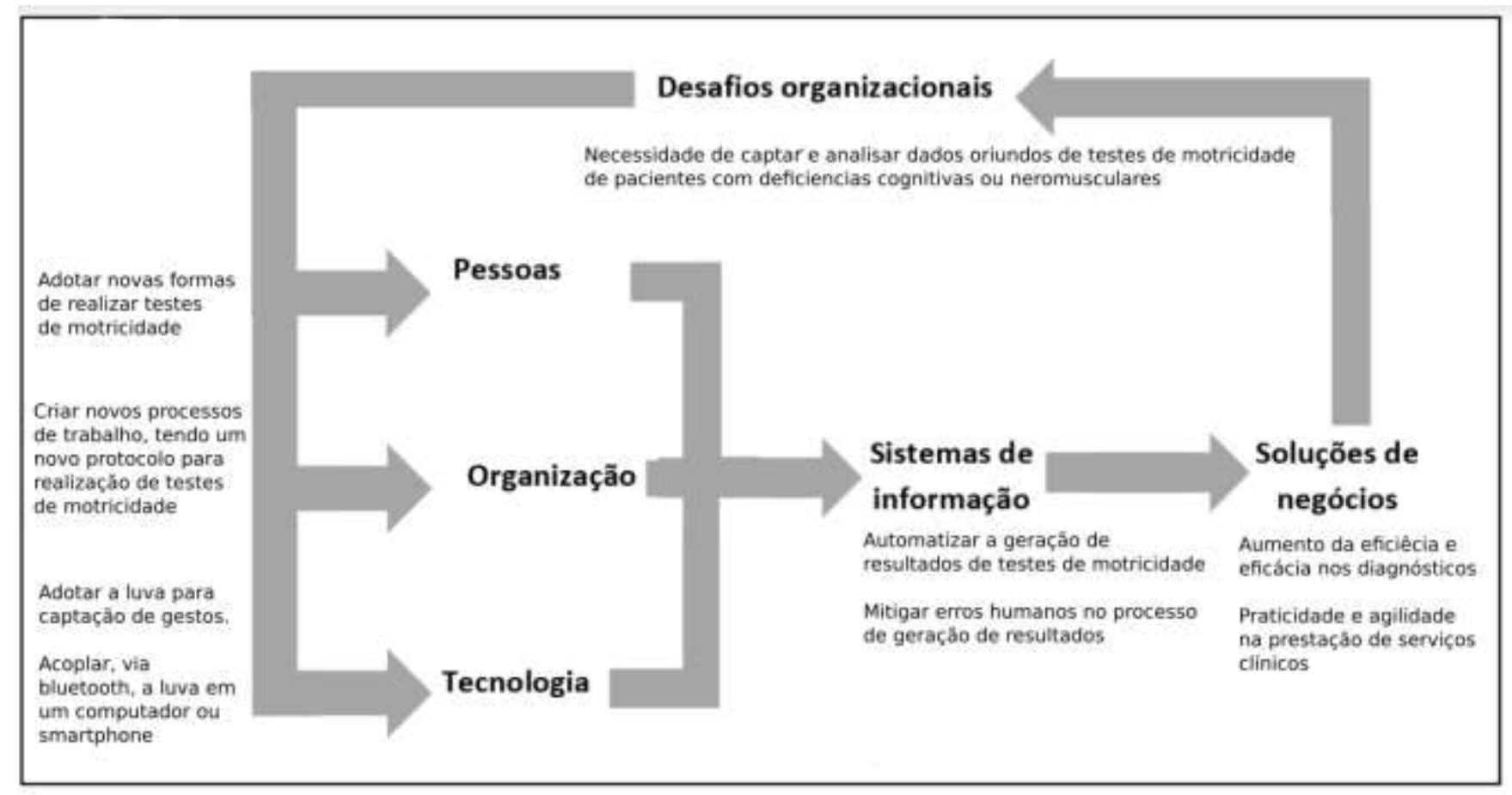

Fonte: Elaborada pelos autores com base no modelo proposto por Laudon e Laudon (2007).

Considerando também as dimensões humanas e organizacionais, verificou-se que a luva de captação de movimentos poderia fazer parte de um sistema de informação que visa automatizar processos e promover maior precisão em testes de motricidade.

A modelagem da versão AS-IS do processo possibilitou analisar também a forma manual de realizar testes de motricidade (sem recursos tecnológicos). Este tipo de teste manual pode envolver anotações em formulários e cronometragem de tempo durante a realização do mesmo.

Na modelagem da versão TO-BE do processo vislumbrou-se como poderia ocorrer a realização de testes de motricidade de modo a automatizar partes do processo, utilizando a luva de captação de movimentos.

Analisando a versão TO-BE do processo observou-se a possibilidade de o sistema ter uma maior precisão no registro dos resultados gerados pelos testes, a exatidão da cronometragem automática do tempo de realização dos testes e o armazenamento automático dos resultados gerados em cada teste de motricidade realizado.

A Figura 4 contém parte da modelagem das versões AS-IS e TO-BE que ilustra como poderia ocorrer um teste de motricidade do tipo labirinto (no qual o usuário precisa conduzir um lápis ou cursor por um trajeto). A versão AS-IS contém a 
simulação de como o teste pode ocorrer manualmente e a versão TO-BE contém a idealização de como o mesmo teste poderia ocorrer utilizando a luva de captação de movimentos.

Figura 4. Parte da modelagem das versões A-IS e do TO-BE do processo de realização do Teste de motricidade fina utilizando a luva de captação de movimentos.

\section{AS-IS: Teste de motricidade fina (labirinto)}

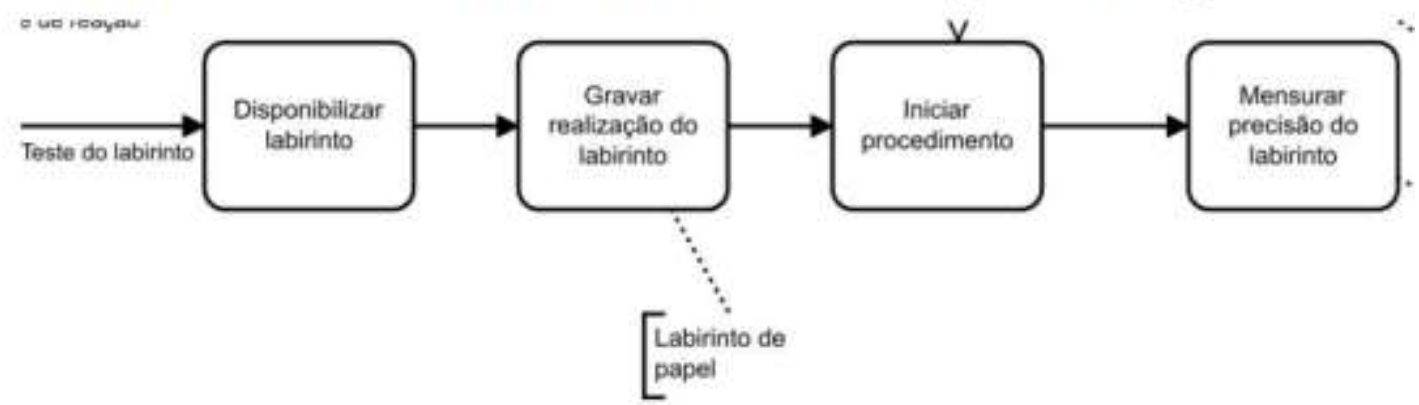

\section{TO-BE: Teste de motricidade fina}

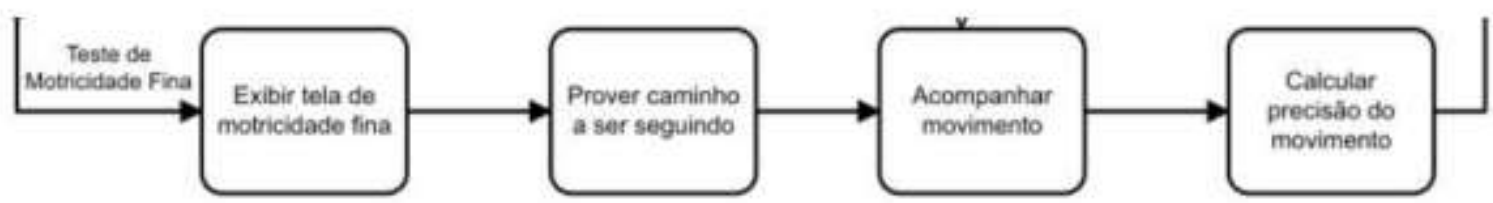

Fonte: Autores.

A modelagem de processos relacionados ao uso da luva de captação de gestos possibilitou verificar a sua aplicabilidade no contexto de soluções de sistemas de informação. A modelagem realizada neste estudo indica que a otimização de processos pode contribuir para o alcance de objetivos organizacionais das instituições prestadoras de serviços de fisioterapia.

No que diz respeito aos resultados obtidos no âmbito do ensino e da aprendizagem de sistemas de informação, pode-se observar que:

- $\quad$ Ensinar e aprender assuntos relacionados a sistemas de informação pode se tornar mais motivador quando for baseado em casos reais;

- Quando o problema ou questão de sistema de informação, que é objeto de estudo, for indicado pelo próprio aluno existe a possibilidade de o mesmo já possuir um conhecimento prévio sobre o assunto, bem como motivadores que envolvam ciclos de aprendizagem significativa.

- $\quad$ A aprendizagem baseada em problemas pode conduzir os alunos a um processo de descoberta de aplicação prática de conceitos teóricos relacionados a sistemas de informação.

O estudo realizado evidencia que a aplicação do modelo de resolução de problemas de sistemas de informação e da técnica de modelagem de processos em um caso real retrata uma situação de ensino e aprendizagem significativa baseada em análise de situações problemas de sistemas de informação.

Apesar do estudo não ter apontado elementos que indiquem que a estratégia de ensino e os recursos didáticos constituem uma abordagem que produz efeitos motivadores mais efetivos do que os de aulas expositivas, cabe mencionar que a referida abordagem vem sendo adotada de modo satisfatória ao longo dos semestres no qual a disciplina é ofertada, tendo-se observado o comprometimento da maioria dos alunos com a resolução de problemas reais de sistemas de informação.

Este estudo não comtemplou uma análise comparativa entre aulas predominantemente expositivas e aulas baseadas na 
resolução de problemas, de modo a comparar o grau de comprometimento e aprendizado de cada abordagem de ensino. Contudo, pode-se inferir que normalmente ocorre um maior comprometimento e a aprendizagem significativa quando os alunos estão envolvidos na resolução de um problema do qual já possui algum conhecimento prévio e estão sendo orientados pelo professor que desempenha o papel de facilitador e motivador.

Além disso, considera-se como indicativo favorável à abordagem adotada o fato de que, após ter cursado a disciplina, parte dos alunos tiveram a iniciativa de publicar, em anais de eventos científicos e em revistas técnico-científica, os resultados do trabalho realizado em aula. Trata-se dos trabalhos como os realizados por Oliveira, Tolfo e Quepfert (2017), Oliveira, Fioravanti e Tolfo (2017), Fernandes, Tolfo e Silva (2018), Chervinski, Tolfo e Milani (2018), Quirino, Girardi e Tolfo (2020).

\section{Conclusão}

Neste artigo apresentou-se um relato de ensino e aprendizagem de sistemas de informação baseado na análise de situações reais como promotor da aprendizagem significativa. A análise da situação-problema apresentada neste estudo envolveu o ensino da proposição de soluções considerando as dimensões humanas, organizacionais e tecnológicas de um sistema de informação. Considerou-se também a perspectiva da melhoria de processos de negócios como parte da solução do problema.

O estudo realizado indica que o modelo de resolução de problemas de sistemas de informação, proposto por Laudon e Laudon (2007), possui aplicabilidade como recurso didático para o ensino e a aprendizagem sobre sistemas de informação em sala de aula. Como recurso didático, o modelo possibilita a análise de situações-problemas considerando as dimensões humanas, organizacionais e tecnológicas presentes em desafios organizacionais que requerem o desenvolvimento de sistemas de informação.

Da mesma forma, o estudo ressalta que a modelagem de processos utilizando a abordagem AS-IS e TO-BE atende os requisitos para ser utilizado como recurso didático na análise de situações-problemas de sistemas de informação. A referida abordagem também possui potencial para ser utilizada na análise de situações-problemas nas mais variadas áreas de conhecimento, visto que a aplicabilidade da visão de processos possui características interdisciplinares e não se restringe aos sistemas de informação.

A estratégia de ensino e os recursos didáticos utilizados caracterizam uma abordagem de aprendizado ativo que pode conduzir à aprendizagem significativa. Contudo, esta afirmação é baseada somente na percepção dos autores, sendo que um deles foi o professor e o outro foi um aluno da disciplina, de forma que não é possível afirmar de modo conclusivo a efetividade da abordagem relatada neste estudo. Ressalta-se que não foram aplicadas técnicas e métodos para verificar a efetividade em questão.

Em virtude disso, trabalhos futuros podem verificar a aplicabilidade da abordagem AS-IS e TO-BE como recurso didático no ensino e na aprendizagem em diferentes áreas de conhecimento, seja em disciplinas das áreas das ciências exatas e da terra, das ciências humanas ou das ciências sociais e aplicadas ou de qualquer outra área em que a perspectiva de processo seja aplicável.

Trabalhos futuros que envolvam o uso de modelo de resolução de problemas de sistemas de informação e a técnica de modelagem de processos como recurso didático devem prever a aplicação de questionários para obter a opinião dos alunos a respeito da estratégia de ensino adotada.

\section{Referências}

Araújo, U. F. D., \& Sastre, G. (2009). Aprendizagem baseada em problemas no ensino superior. Summus.

Ausubel, D. P. (1982). A aprendizagem significativa. Moraes.

Baldam, R., Valle, R., Pereira, H., Hilst, S., Abreu, M., \& Sobral, V. (2007). Gerenciamento de processos de negócios: BPM-Business Process Management. Érica. 
Baldissera, M., Quirino, F. A., Romanssini, M., \& Girardi, A. (2018). Application Development for a Gesture Capture Glove Based on Accelerometers and Gyroscopes. In: Chip in the pampa, 2018, Bento Gonçalves. SFORUM 2018, 2018. v. 18.

Baltzan, P., \& Phillips, A. (2012). Sistemas de informação. AMGH Editora.

Bizagi. (2020). BizAgi Modeler. https://www.bizagi.com/pt/plataforma/modeler.

Braconi, J., \& Oliveira, S. B. D. (2009). Business Process Modeling Notation (BPMN). In: Rogério Valle e Saulo Barbará de Oliveira (Orgs.) Análise e modelagem de processos de negócios: foco na notação BPMN (Business Process Modeling Notation). São Paulo: Atlas. p. 77-93.

Chervinski, J. O. M., Tolfo, C., \& Milani, A. S. (2019). Modelagem de processo aplicada à melhoria da gestão em um Escritório Modelo de Engenharia Civil. Research, Society and Development, 8(12), e178121711-e178121711.

Davenport, T. H. (2014). Big data no trabalho: derrubando mitos e descobrindo oportunidades. Rio de Janeiro: Alta Books.

Fernandes, K. C., Silva, R. P., \& Tolfo, C. (2019). Uma proposta de melhoria de processo utilizando sistemas de informação na gestão de inventário patrimonial. Anais do Salão Internacional de Ensino, Pesquisa e Extensão, 10(2).

Gil, A. C. (2012) Didática do ensino superior. Atlas.

Laudon, C. K., \& Laudon, J. P. (2007). Sistemas de informação gerenciais. Editora Person.

Mancini, A. A. (2005). Aprendizagem significativa: a teoria de David Ausubel. Centauro.

Moreira, M. A. (2010). O que é afinal aprendizagem significativa? 2010. Aula Inaugural do Programa de Pós-Graduação em Ensino de Ciências Naturais, Instituto de Física, Universidade Federal do Mato Grosso, Cuiabá, MT, 23.

Oliveira, A. B. de, Santos Fioravanti, T. dos, \& Tolfo, C. (2018). Proposta de melhoria de um processo acadêmico. Anais do Salão Internacional de Ensino, Pesquisa e Extensão, 10(1).

Oliveira, A. B., Tolfo, C., \& Quepfert, W. E. (2017). Sistemas de informação e modelagem de processos: um relato no ensino. Anais do Salão Internacional de Ensino, Pesquisa e Extensão, 9(1).

Oliveira, D. D. P. (2009). Administração de processos: conceitos, metodologia, práticas. Atlas.

Omg. Object Management Group. (2019). Business Process Model and Notation. < http://www.bpmn.org >.

Pradella, S., Furtado, J. C., \& Kipper, L. M. (2012). Gestão de processos: da teoria à prática. Atlas.

Quirino, F. A., Girardi, A. G., \& Tolfo, C. (2020). Análise de um protótipo de tecnologia assistiva sob a ótica de sistemas de informação. Anais do Salão Internacional de Ensino, Pesquisa e Extensão, 12(2).

Quirino, F. A., Romanssini, M., Girardi, A \& Baldissera, M. (2018). Implementation of Classification Algorithms in a Smart Glove for Hand Gesture Detection. In: Chip in the pampa, 2018, Bento Gonçalves. SFORUM 2018, 2018. v. 18.

Rogers, D. L. (2017). Transformação digital: repensando o seu negócio para a era digital. Autêntica Business.

Romanssini, M., Quirino, F. A., \& Girardi, A (2018). System Implementation of a Gesture Detection Glove for Human-Computer Interaction. In: Chip in the pampa, 2018, Bento Gonçalves. WCAS 2018, 2018. v. 8.

Silveira, D. T., \& Córdava, F. P. (2009). A pesquisa científica. In: Gerhardt, T. E., \& Silveira, D. T. Métodos de pesquisa. Editora UFRGS.

Tolfo, C., \& Della Flora, F. (2016). A gestão de processos de negócio como ferramenta de apoio na gestão da segurança da informação. Revista GEINTEC Gestão, Inovação e Tecnologias, 6(1), 2756-2770.

Tolfo, C. (2017a). Mapas conceituais: aplicações no ensino, pesquisa e extensão. Editora da UFS.

Tolfo, C. (2017b). Aprendizagem baseada em problemas na engenharia de software: relatos de experiências. Ediurcamp.

Tolfo, C. (2019). Modelagem de processos: aplicações no ensino, pesquisa e extensão. Editora UFS.

Tolfo, C. (2020). Aprendizagem baseada em problemas e modelagem de processos no ensino de sistemas de informação. Research, Society and Development, 9(2), e72922087-e72922087.

Tolfo, C., Medeiros, T. S., \& Mombach, J. G. (2013). Modelagem de processos com BPMN em pequenas empresas: um estudo de caso. In: XXXIII Encontro Nacional de Engenharia de Produção - ENEGEP. Salvador, BA, Brasil, 8.

Unipampa. Universidade Federal do Pampa. Curso de Ciência da Computação - Campus Alegrete. (2019). Projeto Pedagógico do Curso Ciência da Computação. http://dspace.unipampa.edu.br/bitstream/riu/97/7/PPC_Ci\%c3\%aancia\%20da\%20Computa\%c3\%a7\%c3\%a3o_versao_2019.pdf.

Valle, R., \& Oliveira, S. B. D. (2009). Análise e modelagem de processos de negócios: foco na notação BPMN (Business Process Modeling Notation). In: Análise e modelagem de processos de negócios: Foco na notação BPMN (Business Process Modeling Notation) (pp. 207-207). 